\title{
Continuous Particle Sampler
}

\author{
Paul B. MacCready, Jr., and Clement J. Todd
}

Atmospheric Research Group, Altadena, Calif.

(Manuscript received 20 February 1964, in revised form 8 April 1964)

\begin{abstract}
A continuous particle sampler has been developed which captures atmospheric particles in a Formvar solvent liquid film. The Formvar solution completely encapsulates the particle; then as the solvent evaporates the film hardens quickly, preserving a replica of the particle. The method yields exact replicas of ice crystal shapes. Liquid droplets are somewhat flattened by surface tension during replication and so a calibration factor is necessary to ascertain the original droplet size from the replica. This calibration factor has been found experimentally by using spores distributed in the droplets to show the volume of the droplet creating each replica. The sampler provides continuous information on particle concentration, particle sizes, and whether the particles are ice or water. The Formvar solution is ordinarily applied to transparent 16-mm movie leader film, and the replicas are viewed by projecting the film with a stop-motion microscope projector.

Several versions of the instrument have been built and used on cloud physics research aircraft. Special adaptations have been made for ground use: to sample fogs, to measure crystals in a freezing nuclei counter, and to collect snowfiakes.

Various design compromises are required in the operational instruments to overcome, over a broad range of meteorological conditions, problems such as those associated with film coating, droplet encapsulation, droplet migration and spurious crystal growth. These factors and others associated with particle collection, data reduction and interpretation are discussed. Examples of the field use of the sampler are given.
\end{abstract}

\section{Introduction}

The continuous particle sampler was developed primarily to provide a method for ascertaining quantitatively the coexistence of ice crystals and supercooled water droplets in a cloud being seeded by natural or artificial freezing nuclei. It has turned out to handle this job particularly well, and also perform other valuable related tasks.

The device is a continuous version of the Formvar ${ }^{1}$ method developed by Schaefer (1956) for making ice crystal replicas. Its main virtue is that it makes permanent replicas of the shapes of particles which impinge on a liquid-plastic surface. The Formvar plastic solution flows over the particle, completely enclosing it, and then the solution hardens as the solvent evaporates. The encapsulated particle may then evaporate or sublimate away, but its replica is preserved.

In the airborne continuous sampler, the liquid-plastic Formvar-solvent film is put on transparent 16-mm film which is continuously drawn past a slot exposed to the ambient airflow. The Formvar solution then hardens before the exposed portion reaches the take-up reel. Conceptually, the instrument is simple. However, the practical instrument requires that many design compromises be made to give proper coating, proper hardening, and good particle collection and replication.

1 Trade name, Formvar 15-95E, Shawinigan Resins Corp., Springfield, Mass.
The active development of this sampling method has been proceeding for three years, through both the Atmospheric Research Group and Meteorology Research, Inc., (MRI), with sponsorship by the National Science Foundation, the Army Electronic Research and Development Laboratory ${ }^{2}$ and the Naval Research Laboratory, ${ }^{8}$ as well as MRI. This paper reviews the development, shows the present state of the technique, and considers the numerous interrelated factors on which the technique depends. It is felt that the method has general applicability for studies in which knowledge of the size spectra and particle types of aerosols are needed.

2 A first final report "Study and Modification of Convective Storms," by P. B. MacCready, Jr., T. B. Smith, C. J. Todd, C.-W. Chien and E. Woodward, Meteorology Research, Inc., to Army Electronic Research and Development Laboratory, Ft. Monmouth, N. J., Contract No. DA 36-039 SC-89066, 1963, gives some details about the sampler and data obtained with it during the summer of 1962. Further discussions of the sampler and data processing, and recent examples of the quantitative use of the instrument in obtaining droplet spectra in rising cumulus cores, are given in "Study and Modification of Convective Storms," by P. B. MacCready, Jr., T. B. Smith, C. J. Todd, A. I. Weinstein, 1964; Final Report, Part B, Report No. 4B, by Meteorology Research, Inc., to U. S. Army Electronic Research and Development Laboratory, Ft. Monmouth, N. J., Contract DA 36-039 SC-89066.

8Final report "Continuous Particle Sampler Study Program," by P. B. MacCready, Jr., and R. E. Williamson, Meteorology Research, Inc., NRI-ONR Contract Nonr-3819(00)(X), December 1963, gives details on many of the items reviewed in this paper, and describes the latest version of the sampler. 


\section{Background}

In 1961, trials with various croplet collection methods showed that Schaefer's Formvar replication technique could lead toward an operational continuous sampler. An important factor in the development of the system has been the availability of a $16-\mathrm{mm} \mathrm{film}^{4}$ which would not dissolve in the solvents used (chloroform or ethylene dichloride). This meant that $16-\mathrm{mm}$ film handling methods could be adapted to the sampler, which was a great convenience for the drive mechanism as well as in the subsequent viewing of the replicas.

The first complete sampler system was used successfully in the summer of 1961 on the Atmospheric Research Group Flagstaff studies. In this dipper tank system the Formvar coating was precoated on the leader film, and then softened before exposure by running the film through a solvent bath maintained at a particular level by means of a float valve. System design and some results were presented by Todd (1961).

In the fall of 1961 the unit was taken to a droplet collector comparison conference in France organized by H. Dessens. A miniature wind tunnel, consisting of a

"Cronar, P40A Leader, Polyester Photographic Film Base, duPont, Wilmington, Delaware.

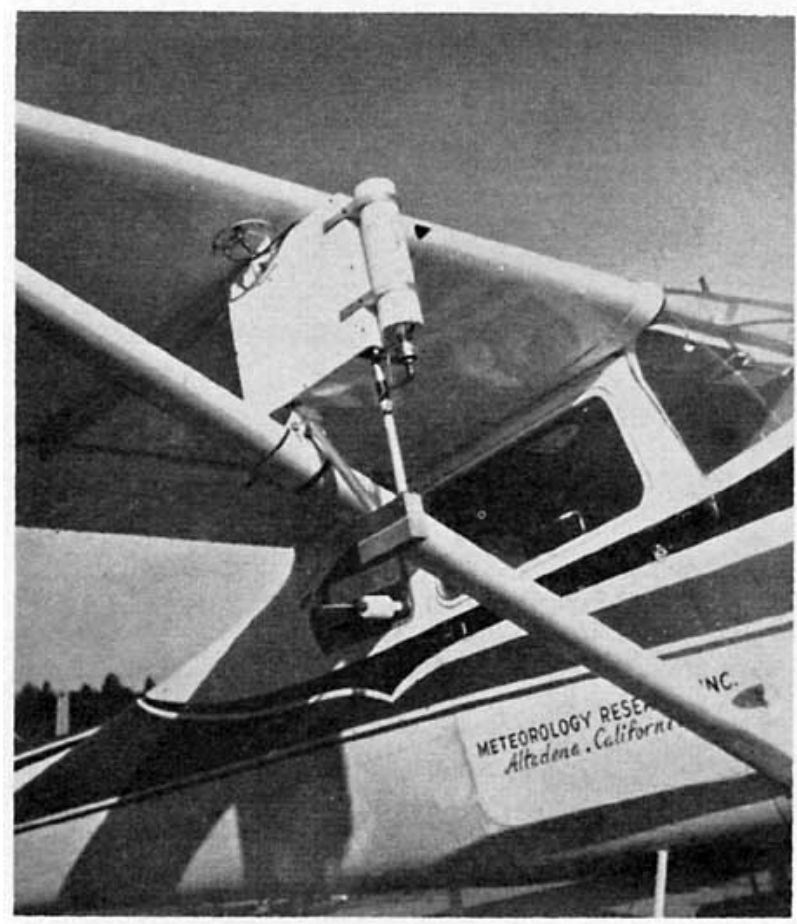

Frg. 1. Coating wheel version mounted on a Cessna 180. The tank at the bottom contains the Formvar-solvent solution and applies it to the film via the coater wheel. The cylindrical tank above is not used; it was only employed for the dipper tank version. The drying tubes can be seen extending under the wing to give extra drying time before the film reaches the take-up reel. The lips around the exposure slot are heated for de-icing. The supply and take-up reels are both mounted on one shaft. tube and a blower, provided the air flow on the ground to substitute for the airplane motion. This device was operated satisfactorily in-cloud on a mountain peak, along with collector equipment of other investigators. The results were given by MacCready (1962).

In the summer of 1962, two improved units were installed on two aircraft, one unit being the precoated film variety, the other using uncoated film and applying the Formvar-solvent mixture just before exposure by means of a coating wheel (see Fig. 1). The coating wheel method seemed the more practical (more controllable, and less likely to stick), and an adaptation of it was used for the 1963 summer measurements (see Fig. 2). Research results for the summer studies obtained with the device have been presented in the MRI report of Footnote 2 and by Todd (1964). Still another coating wheel version (Figs. 3 and 4 ) was developed in the fall of 1963, utilizing the knowledge obtained from the field studies with the previous units.

Hindman (1964) has presented two versions of continuous sampling by the Formvar method for particles impacting by gravity settling. For one version the particles collected were ice crystals grown from freezing

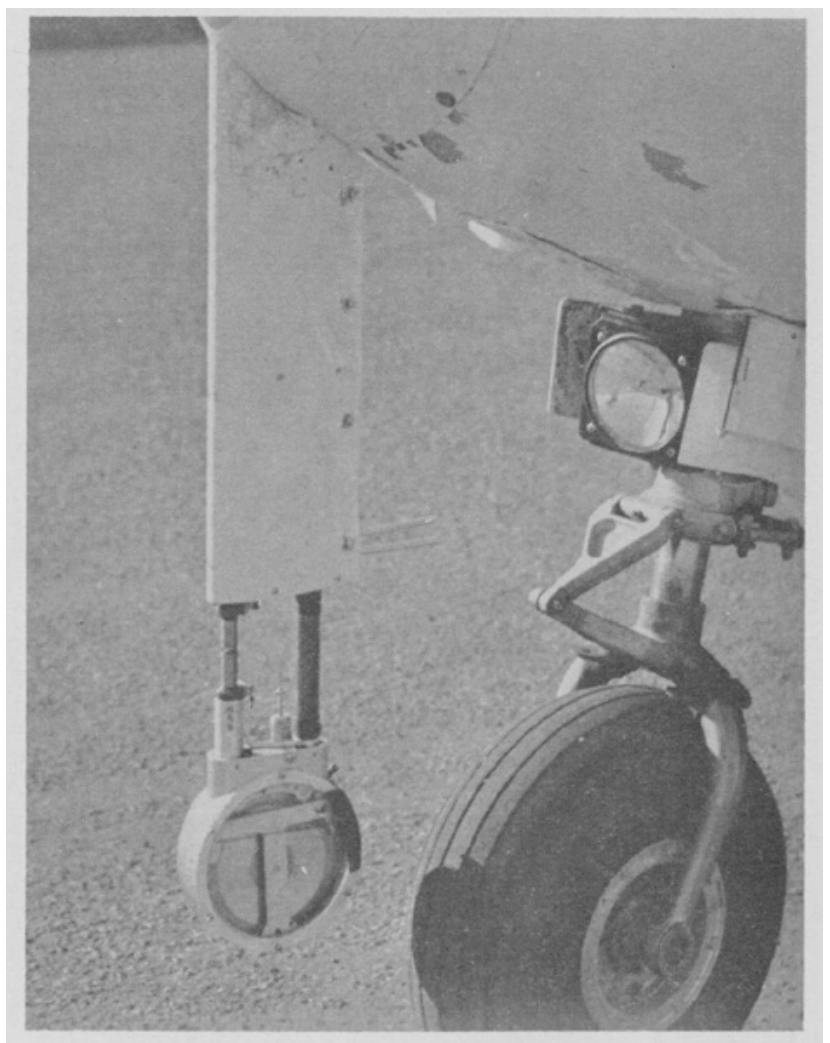

FIG. 2. Coating wheel system installed in the nose of a twin engine Apache aircraft. The drying tubes leading the film from exposure area to take-up reel were electrically heated to stop blushing and spurious crystal growth, and, around the slot, to prevent icing. The drying tubes went into the cockpit area, where a projector showed the replicas on a small screen so as to permit in-flight evaluation. 


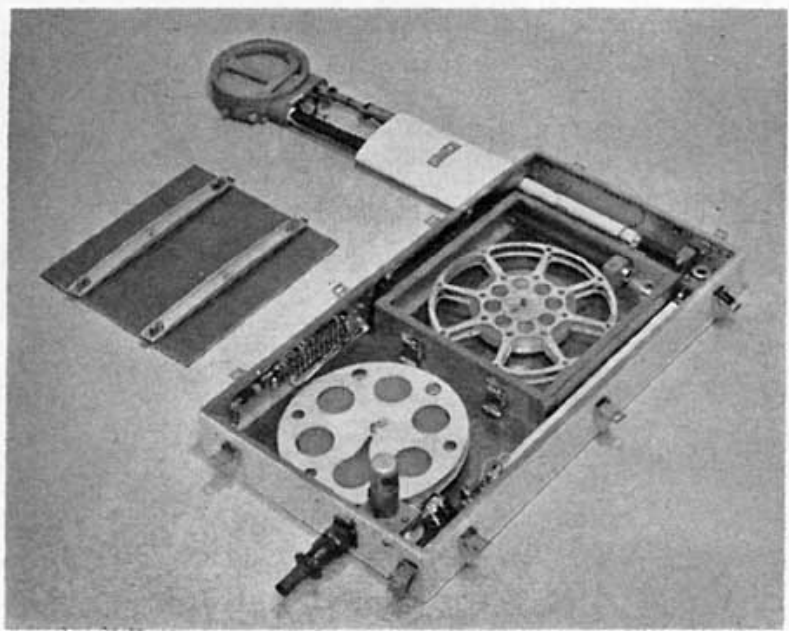

FIG. 3. Latest continuous sampler. This uses the coating wheel principle and heated drying tubes. The supply reel compartment is sealed tightly to prevent air backflow down from the slot which could preharden the coating and also permit moisture from the air to contaminate the tank.

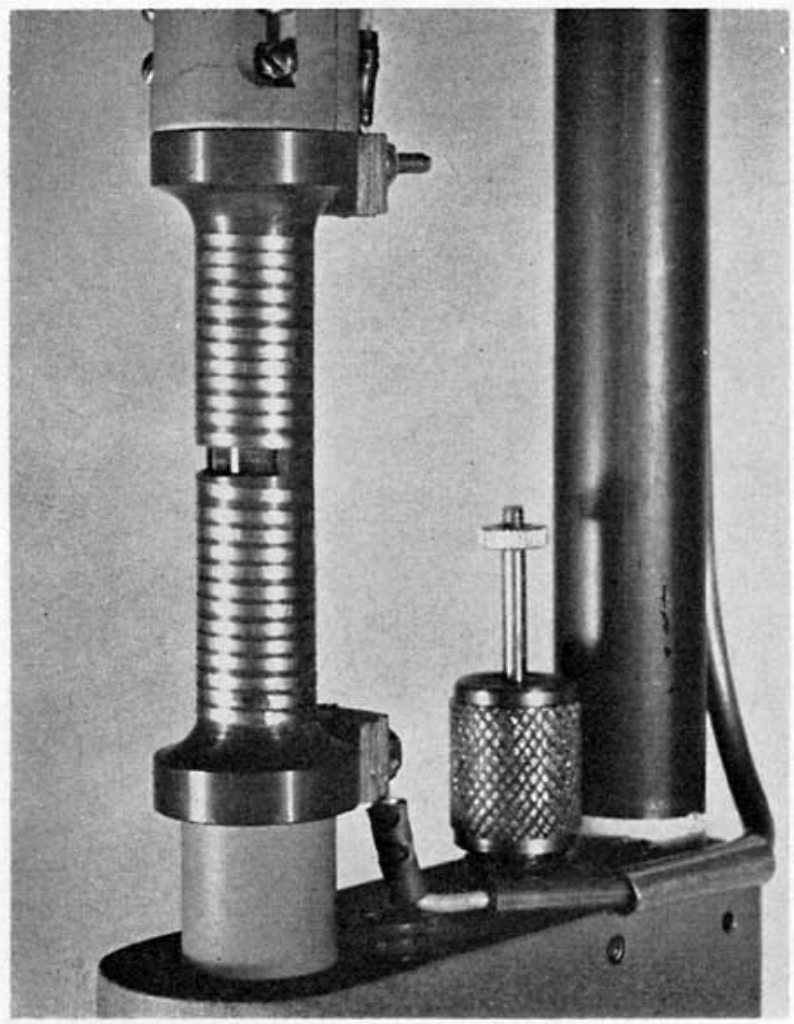

FIG. 4. Detail of the window showing the de-icing wire.

nuclei in a continuous cold box, while in the other case the particles were tiny hydrometeors falling from the ambient air. In either case the particles land on a dry Formvar coating which is subsequently softened during passage through a chamber with chloroform vapor. This is a continuous analog of the vapor replication technique investigated by Schaefer (1962).

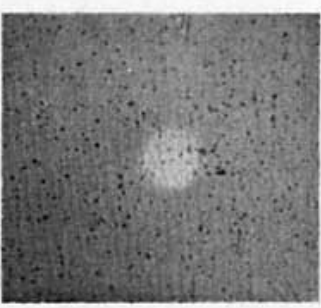

a

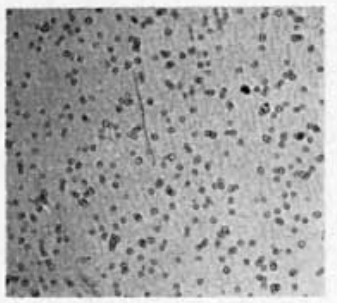

C

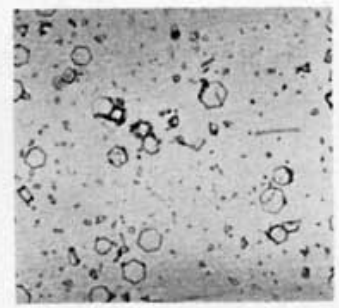

e

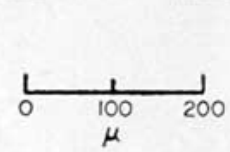

FIG. 5. Replicas during spiral ascent in the core of a cumulus cloud being seeded with silver iodide, I lagstaff, 15 August 1962 . a. $5,720 \mathrm{~m},-9.8 \mathrm{C}$. Just at cloud base. Small droplets approximately $3 \mu$ diameter. b. $5.735 \mathrm{~m},-10$.()C. Droplets larger, $5-7 \mu$, $12 \mu$ ice crystal. c. $5,795 \mathrm{~m},-10.3 \mathrm{C}$. Jroplets to $9 \mu$. Unseeded portion, no crystals. d. $5,870 \mathrm{~m},-11$.(1)C. Crystals and smaller droplets. e. $6,405 \mathrm{~m},-13.9 \mathrm{C}$. Hexagonill, square, and triangular plates, with small droplets. f. 6,618.5 m, -15.4C. Large cracked and broken crystals.

Fig. 5 gives some representative results of the use of the continuous sampler. Most experience with the technique so far has been derived in summer at Flagstaff, Ariz., in cumulus clouds with rather cold bases.

\section{Design factors and compromises}

The Formvar coating on the filın must be soft during the impaction process, and flow readily so as to encapsulate the particle, but still be stiff enough to resist being blown off by the wind, and stiff enough to keep the droplets from clumping together (here called flocculation). The coating hardens as the solvent evaporates. This hardening must not take olace before the film passes the slot, but must occur before the film reaches the take-up reel and before the purticle size or shape is significantly altered. The evaporation cools the film, 
and water from the ambient air can condense on the film (here called blushing) or can cause spurious growth of ice crystals. This cooling effect can be eliminated by adding heat to the system, but excessive heat can melt the ice crystal or even evaporate water droplets before replication is complete. These interrelated factors require careful design compromises. The task gets harder as the range of operating conditions is increased to cover higher air speeds and colder temperatures, and the task is further complicated by mechanical problems such as film sticking and elongation of sprocket holes.

Mechanical coating considerations. The first continuous cloud particle collector used a method in which the film was precoated and dried in the laboratory, and then the hardened coating was softened by immersion in the solvent dipper tank just before the film was exposed to the air stream. The coating in the laboratory was accomplished with a broad ( $6 \mathrm{~mm}$ wide) slit pen, siphon fed from the Formvar solution supply bottle. It was found that if a solution of at least 6 per cent Formvar was used the coating would not orange-peel or wrinkle during the drying process. In general the thicker solution produced smoother and more uniform coatings.

Considerable effort was expended in attempting to develop a continuous applicator using a wick, but the results were not encouraging. The wick or pad always left a streaked surface, large feed pressures were required in order to produce acceptable coating rates, and the method involved numerous operational difficulties.

The roll-on coating wheel (Figs. 1-4) proved to be reliable operationally. In the coating wheel method the moving film is pressed against the top edge of a thin, large diameter wheel causing it to rotate. The bottom portion of a wheel lies in a reservoir of the coating solution, and as the wheel rotates a stripe of this solution is transferred to the film at the point of contact. In practice the edge of this wheel, which is about $3 \mathrm{~mm}$ wide, is machined to form a trench on the order of $300 \mu$ deep. In order to minimize the deposition of excess Formvar at the edges of the stripe, an adjustable scraper was fitted. This scraper consists of thin, stainless steel blades which are in contact with the perimeter and sides of the wheel and serve to wipe off any excess solution which adheres to these surfaces.

The vapor pressure of the solvents used is very high and any flow of air over the solution surface causes rapid evaporation. To prevent a flow of unsaturated air over the solution, the tank and film magazine portion of the system should be pressurized or sealed so that their atmospheres are in equilibrium with the stagnation pressure (see Fig. 3). This configuration also minimizes hardening of the Formvar solution and evaporative cooling prior to exposure at the window slot.

Coating faclors. Both chloroform $\left(\mathrm{CHCl}_{3}\right)$ and ethylene dichloride $\left(\mathrm{C}_{2} \mathrm{H}_{4} \mathrm{Cl}_{2}\right)$, as well as mixtures of both, have been used as solvents for the Formvar. The chloroform is the more volatile solvent, by a factor of $2 \frac{1}{2}$ or 3 .
The melting point of chloroform is $-63.5 \mathrm{C}$, while it is only $-35.5 \mathrm{C}$ for ethylene dichloride, and thus the use of chloroform is indicated at cold temperatures. Replication with a continuous sampler at $-43 \mathrm{C}$ has been achieved. The rapid cooling by solvent evaporation during Formvar hardening can be severe with chloroform, and so sometimes the use of ethylene dichloride is preferable.

Ice crystals seem to replicate best with a thin film (minimizing evaporative cooling and making the regulation of heat less critical) and dilute Formvar concentration (which gives the best replica resolution). Such a thin film tends to magnify troubles of skinning over the film before reaching the impaction area, which can have a bad effect on droplet replication and, to a lesser extent, on crystal replication. The conditions which are best for ice crystal replicas tend to permit flocculation of water droplets. Thick concentrations minimize flocculation. Thick film provides less droplet distortion but tends to blow off more in the air stream, especially when of dilute concentration.

It appears from flight tests that, with adequate drying heat, a concentration of between 2.5 per cent and 4 per cent of either solvent in the coater tank is about optimum. When the concentration is less than 2.5 per cent the air stream displaces the coating at relatively low air speeds, except for very thin films (as was the case for Fig. 5). When the concentration is greater than 4 per cent the replication efficiency appears to decrease.

There seems to be a reasonably broad range of film thickness and concentrations for good droplet collection and replication, or alternatively for good ice crystal collection and replication, with either chloroform or ethylene dichloride. The ranges are extended with careful control of drying heat. There is a much more limited range of film thickness and concentration for good simultaneous collection and replication of coexisting droplets and crystals. Both droplets and crystals have been obtained at once with thin 1 per cent ethylene dichloride (used for samples on Fig. 5), and on other occasions with thicker film of $2 \frac{1}{2}$ per cent chloroform $(65$ per cent) and ethylene dichloride (35 per cent) mixture. More field tests are required to show if the ranges for collection and replication of both droplets and crystals are so critical that a dual instrument would be needed for reliable field investigations.

Neither the drive motor nor the rest of the electrical system may be considered spark-proof, and therefore, in order to eliminate the possibility of what could be quite a violent explosion, it is recommended that a noninflammable solvent such as chloroform be used rather than the ethylene dichloride. Since the chlorinated hydrocarbons and in particular chloroform are toxic and their effect is to some extent cumulative, the equipment should be mounted and vented in such a way that personnel are not exposed to sensible concentrations of vapor. 
Blushing and ice crystal growth. Blushing and spurious ice crystal growth can be completely controlled by using heat at the window and along the drying tubes. Even without heat, very thin films have provided good crystal replicas because the total amount of solvent causing evaporative cooling is small. The particles put on the film by blushing are small, on the order of 1 or $2 \mu$, and often irregularly shaped. The larger droplet replicas can be evaluated even in the presence of blushing except in severe cases. Blushing is not a problem when droplets are replicated with Formvar solution on a glass slide, presumably because the thermal inertia of the slide keeps the temperature up. This thermal inertia effect is not easy to apply in the continuous sampler.

Flocculation. Flocculation is the term used to describe the adhesion of several droplets to one another beneath the Formvar film. Flocculation has been most frequently observed when thin, low percentage solutions are employed, and when the droplet diameters are greater than the thickness of the coating. Considerable flocculation can occur apparently without coalescence, and so the droplet size and concentration information is not lost by flocculation.

High aircraft speeds. The coater system replicated reliably at speeds up to $80 \mathrm{~m}$ per sec. High speed can result in partially blowing off the liquid film; speed is also sometimes associated with the presence of bubbles in the film; and speed intensifies the problem of film hardening prior to reaching the exposure slot. All these effects are minimized by the use of a narrow (in the film movement direction) window slot rather than a wide one. The slot width and film speed (often 5 to $20 \mathrm{~cm}$ $\mathrm{sec}^{-1}$ ) are adjusted to keep the number of droplets collected, in clouds with the maximum liquid water contents, down to where flocculation or overlapping of droplets is statistically insignificant. It has proved advantageous to seal the supply reel to prevent large air flows from the slot along the tube toward the supply reel (see Fig. 3). This minimizes prehardening of the film, and avoids putting contaminants from the ambient air in the coater tank. For high speed aircraft it is likely that the best technique for sampling would be to introduce a diffuser to slow the flow at the window slot, although this would complicate the collection efficiency calculations.

\section{Absorption and evaporation of the vapor}

From the foregoing discussions it is apparent that vapor softening of a precoated Formvar film might be desirable, to permit more quantitative control of the film characteristics and to avoid the complexities of dipping or coating the film in flight. Therefore simple tests were conducted to provide some data on the rate at which precoated film can be softened by vapor. Chloroform vapor was used because it has a much higher vapor pressure than ethylene dichloride at any given temperature. The same laboratory setup used for the absorption tests served to give information on the evaporation rate of the chloroform from the Formvarchloroform film.

A short piece of film with a pre:soated Formvar strip on it was suspended from a sensitive analytical balance down in a cold chamber at $-20 \mathrm{C}$. A vapor chamber (a container lined with chloroform-;oaked blotters) was kept at a given warmer temperature, say $-11 \mathrm{C}$, and then placed around the film. The rate at which vapor was absorbed was noted. For the evaporation tests the vapor chamber was withdrawn and the weight decrease noted. as a function of time. Fig. $t$ gives the absorption results for a thin coating about $2 u$ thick. It is evident that the absorption takes place ir two regimes: a) the first 30 seconds, during which the rate depends almost directly on the initial temperature difference between film and ambient air, and $b$ ) the priod after 30 seconds when the rate is slow and is rather constant no manner what the starting temperature. During period (a) presumably the film is much cooler than ambient. During period (b) presumably the film temperature is close to the vapor chamber ambient temperature.

The results point out that the rapid absorption of vapor will take place only if the rapor is substantially warmer than the film on which it is to condense. Extrapolating from the figure, it is possi jle that dry Formvar $1 \mu$ thick could be softened sufficiently in 30 seconds by the vapor method if the vapor-filr a temperature difference were kept at $30 \mathrm{C}$. Thus the rapor softening technique may be feasible for a contir.uous sampler, but it will not be particularly convenient.

The tests showed that the initic.l absorption rate for $5 \mu$ film is about the same as for the $1 \mu$ film, although both eventually will reach the same concentration with

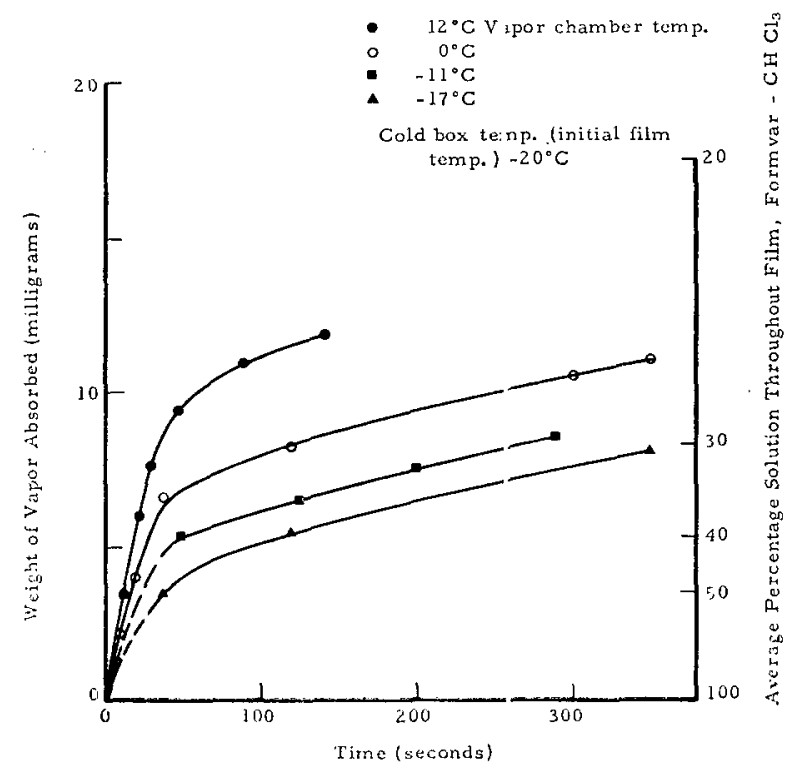

FIG. 6. $\mathrm{CHCl}_{3}$ vapor absorption into Formvar. 
the thick film absorbing more solvent. Thus the thinner film will reach a particular softness much more quickly than will the thicker film.

The evaporation rate test results proved to be virtually mirror images of the absorption tests. They thus verified that thin film will stiffen much more quickly than thick film. The data suggest that the vapor pressure of the ambient air must be kept low (presumably by ventilation) when rapid evaporation is desired.

\section{Droplet distortion}

When an ice crystal impacts on the softened Formvar film, the subsequent replica is a perfect, hollow, threedimensional casting of the crystal in the Formvar. If the impacting particle is a liquid droplet, the situation is much more complex because the droplet will distort. The Formvar will still encapsulate the droplet (probably in a millisecond for small droplets), the solvent will evaporate and leave a Formvar replica, and the droplet will eventually disappear by evaporating through the thin Formvar skin. However, the exact shape the droplet assumes will depend on the original spherical droplet diameter, the thickness of the Formvar coating, and the surface tensions of the Formvar solution to air and to water. As the solvent evaporates, the Formvar coating thickness changes, and the surface tensions change as the Formvar solution gets denser (and the solution concentration will get denser more quickly in the thin skin over the droplet than in the main film). These varying factors will keep readjusting the droplet shape until the Formvar solution viscosity increases to the point where no further shape change will take place (the Formvar gets so viscous it is effectively solid). For the practical utilization of the continuous sampler it is essential to establish the calibration factor, replica radius divided by original droplet radius, for various conditions of film thickness, film material, and droplet size.

In the foregoing it has been assumed that the droplet and solvent do not merge with each other. Actually the solvent may dissolve in the water to the extent of about 1 per cent of the water mass. Thus an indeterminacy in true original droplet mass car arise, but the amount is negligible compared to other errors associated with the measurement and correction of droplet diameters.

A mathematical calculation of the distortion of the droplet was made, by equating surface tension energies, based on a very simple picture: the droplet, originally spherical with radius $r$, falls in $\approx 0$ a Formvar solution of thickness $h$, and is distorted to an elliptical cross section with semi-major axis $a$ and semi-minor axis $b$; the Formvar skin or cap which coats over the top of the droplet has radius $c$. These rela:ionships are shown in Fig. 7. In the actual case the droplet is flattened but not in such an ideal oblate spheroid shape, and the Formvar solution and Formvar cap skin have varying strengths as the solvent evaporates.

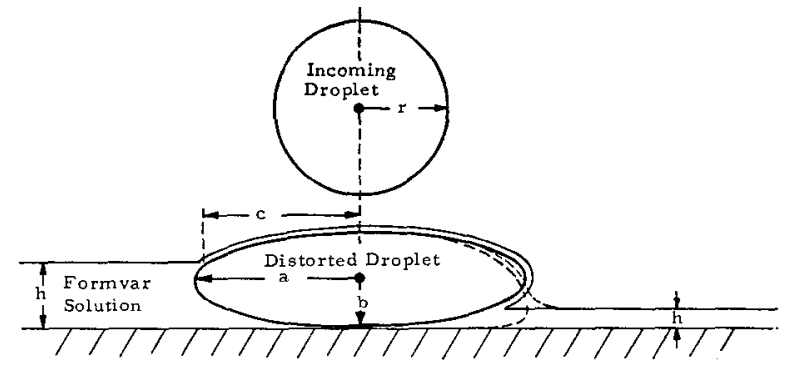

FIG. 7. Simplified model of the distorted droplet. The left side of the picture gives the case for $h>b$, while the right side of the picture gives the case for $h<b$. For $h>2 r$ the droplet is entirely beneath the flat Formvar surface, $c=0$, and the droplet remains spherical so $a=b=r$. The dashed lines show a more realistic shape of the droplet and Formvar solution, but a shape which is more difficult to treat mathematically.

When the spherical droplet, with surface area $S_{S}$ is distorted into the oblate spheroid shape, its area will be increased to $S_{E}$ and energy

$$
\gamma_{F / W}\left(S_{E}-S_{S}\right)
$$

will have to be added. $\gamma_{F / W}$ refers to the surface tension of the Formvar solution with respect to water. The energy available to cause this distortion is considered derived from raising the Formvar skin which flows over the top of the droplet. The film which originally had area $A_{c}$ is distorted into a cap which has area $S_{T}$, and so the energy is

$$
\gamma_{F / A}\left(S_{T}-A_{c}\right)
$$

$\gamma_{F / A}$ refers to the surface tension of the Formvar solution with respect to air.

Equating the energies,

$$
\frac{\gamma_{F / A}}{\gamma_{F / W}}=\frac{S_{E}-S_{S}}{S_{T^{-}}-A_{c}} .
$$

The ratio $\gamma_{F / A} / \gamma_{F / W}$ was measured for some Formvarchloroform solutions. It reaches a peak of 1.4 at 2 per cent, and decreases to 0.4 at 10 per cent; it is presumed to be somewhat lower at the more pertinent higher concentrations. $\left(S_{E}-S_{S}\right) /\left(S_{T}-A_{c}\right)$ was computed as a function of the desired $r$ and the measurable $h$ and $a$. A computer assisted in the rather complex calculations required. The results cannot be deemed quantitatively informative, due to the oversimplification of the picture on which they are based, as well as due to a lack of accurate knowledge of the $\gamma_{F / A} / \gamma_{F / W}$ ratio for very thick solutions. Nevertheless, the calculations do show that the correction factor $a / r$ does depend on the surface tension ratios $\gamma_{F / A} / \gamma_{F / W}$ but that for normal values of $\gamma_{F / A} / \gamma_{F / W}$ the dependence is not very strong. The computations also served to assist in understanding the results of the absolute calibration study. 
The method chosen for a rough absolute calibration was an adaptation of the one employed by Farlow and French (1956). It provides for the simple and rapid determination of micro-drop volumes by the inclusion of uniform size solid particles in the water drops. The principle involves the use of a suspension, in water, of solid particles of uniform size. If the suspension is vigorously stirred, any small droplet will contain the same concentration of particles as the suspension from which it originated, and so the original droplet volume can be estimated from the number of particles observed in the replica.

Several types of inorganic particles were tried, but they either dissolved or had too broad a size spectrum. A survey of available natural particles led to the selection of the spores of the common mold, aspergillus niger. The density of the particles is greater than that of water but the particles are practically insoluble in chloroform-Formvar solutions. The particles were quite uniform with a diameter of about $3 \mu$. The spores were shaken from the mold and placed in suspension in distilled water. The stock solution was vigorously stirred and maintained in the bath of an ultrasonic cleaner. Some of the stock solution was aspirated at the window of the coater system equipment, with an air speed of about 35 meters $\mathrm{sec}^{-1}$. Because of the small quantity of the stock solution only two test runs were possible, one run on a solution of 3.5 per cent Formvarchloroform and one on 6 per cent Formvar-chloroform. The thickness of the dried coating of Formvar $\left(h_{f}\right)$ from these two runs was arrived at by averaging many readings of a micrometer screw gauge and also by deducing the thickness by gravimetric procedures $(10 \mu$ for the 3.5 per cent solution, $23 \mu$ for the 6 per cent solution). The original volume of the droplet was taken to include that occupied by the particles. A total of 320 counts were made for calibration of the stock solution. The concentration of particles per unit volume in the stock solution was determined by means of a hemacytometer. Somewhat over a hundred counts were made for calculation of replica droplet volume. Counts were averaged for replicas having the same diameter. The small number of replicas counted was due to the poor yield of usable sizes stemming from the crudeness of the aspiration equipment and the small volume of calibration suspension produced. The probable error for computations of volume from single points on the presented curves must be considered to be slightly over 20 per cent based upon the mean deviations of the experimental data, however the apparently good fit of the curves indicate that this might be pessimistic. The results are presented on Fig. 8.

Consideration of Fig. 8 shows an important point, namely, that the curve for the 6 per cent solution and the curve for the 3.5 per cent solution are about the same when plotted in terms of $r / h_{f}$ or $a / h_{j}$. Such a plot has been made as Fig. 9. As would be predicted from the model discussed previously, for $a / h_{f}<0.5$, the droplet is entirely below the surface and $s$ undistorted, $a / r=1$. For larger droplets there is distortion and $a / r>1$. Both the 6 per cent curve and the 3.5 per cent curve are the same, to within the accuracy of the measurements on which they are based. One would hypothesize that, as a reasonable first approximation, tine same relation holds for other Formvar solution concentrations.

The curves for various concentrations will be a unique function of $a / h_{f}$ only if the original wet solution thickness is of no importance. Apparently the final shape of the droplets is determined by droplet size and the Formvar solution details after the solution has evaporated until it is considerably more concentrated than 6 per cent. Therefore the initial concentration is of no concem (except for other problems such as film coating, complete encapsulation, flocculaition, blushing, etc.).

When the calibration factors shown on Fig. 8 were examined with respect to the theoretical computations on distortion, another factor becıme evident. For thick films, where $a / h_{f}$ is not much larger than unity, the final distortion is in agreement with theory for rather low $\gamma_{F / A} / \gamma_{F / W}$ ratios-ratios which imply a very thick solution from which almost all the solvent must have evaporated. This will of course co ne about because until the concentration of the evaporating film is thick, the film thickness will exceed the sijherical droplet diameter. For thin films (or large droplets) where $a / h_{f}$ is large, the final distortion is in agreement with theory for higher $\gamma_{F / A} / \gamma_{F / W}$ ratios - ratios which imply a rather thinner solution. It is presumed in this second case that the Formvar cap gets stiff, from evaporation, rather quickly; thus the shape and henise the calibration ratio $a / r$ is determined by the stiff cap while the main solvent film is still not very stiff.

The mean curve of Fig. 9 can be considered as the basic calibration for chloroform sulutions of any starting concentration. In a practical case, $h_{f}$ can be measured from the film and then Fig. 9 used to find $r$ for the values of $a$ encountered. In actual use, $h_{f}$ will be found to be rather constant, and so from Fig. 9 a family of curves resembling those of Fig. 8 can be derived. From this plot, $r$ can be read off directly from $a$ on the appropriate concentration line.

The absolute calibration ha: been done only for chloroform solutions. For ethylene dichloride, one calibration is available from MacCready (1962). For the droplets studied, $a=15 \mu$, and $r=10 \mu, a / r=1.5$. The film thickness was estimated at $10 \mu$. If it had been $6 \mu$, this point would fit perfectly on Fig. 9. The ethylene dichloride would be expected to have a somewhat different calibration than the chloroform because of the differing surface tension ratios, but the theoretical calculations imply that the effect should not be large.

For very thin films the droplet distortion should vary only slightly with film thickness. This is consistent with the physical picture shown by the dashed curve in Fig. 7 . Observations of replicas on a film with varying thickness (from uneven coating) implied that the replica size at 
the thinnest portions was not much greater than that where the film was thicker. This would suggest that $a / r$ does not increase indefinitely with $a / h_{f}$ on Fig. 9, perhaps reaching a limit below 3 .

\section{Size ranges}

The small end of the size range of particles which can be treated by this sampler depends essentially just on collection efficiency. In all the versions of the continuous sampler, as shown in Figs. 1 through 4 , the film is curved around a longitudinal axis and fits snugly behind the slot. Thus to a first approximation the collecting surface represents a section of a circular cylinder, and so its collection efficiency can be computed from the curves presented by Langmuir and Blodgett. ${ }^{5}$

To illustrate typical values, Fig. 10 shows the collection efficiencies computed for conditions which were representative of those on the Flagstaff cumulus studies. The curve for the stagnation point of the $1.2 \mathrm{~cm}$ diameter cylinder is one actually used in data reduction; the other two curves demonstrate the differences caused by using a larger cylinder or considering the whole cylinder rather than the stagnation point. Normally the particles

${ }_{5}^{5}$ Langmuir, I., and K. B. Blodgett, 1945: Mathematical investigation of water droplet trajectories. General Electric Co. Report No. RL-225, Final Report Army Contract W-33-038-ac9151.

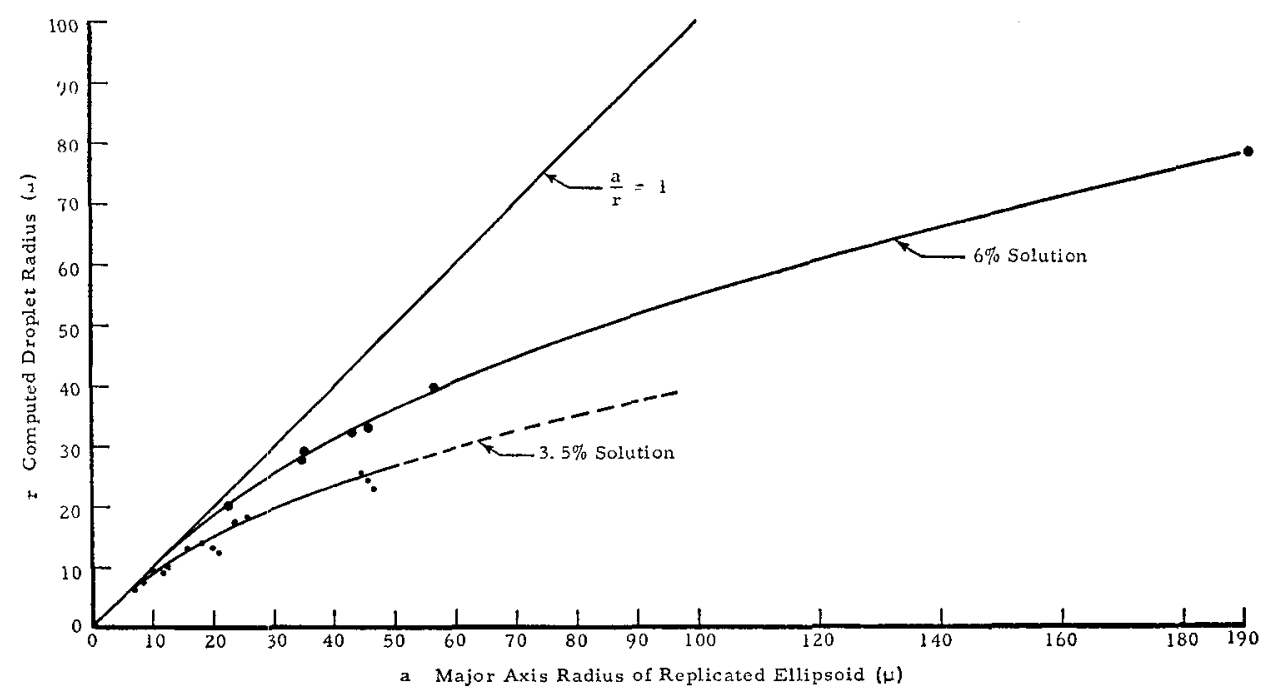

FIG. 8. Distortion calibration with aspergillus niger spores.

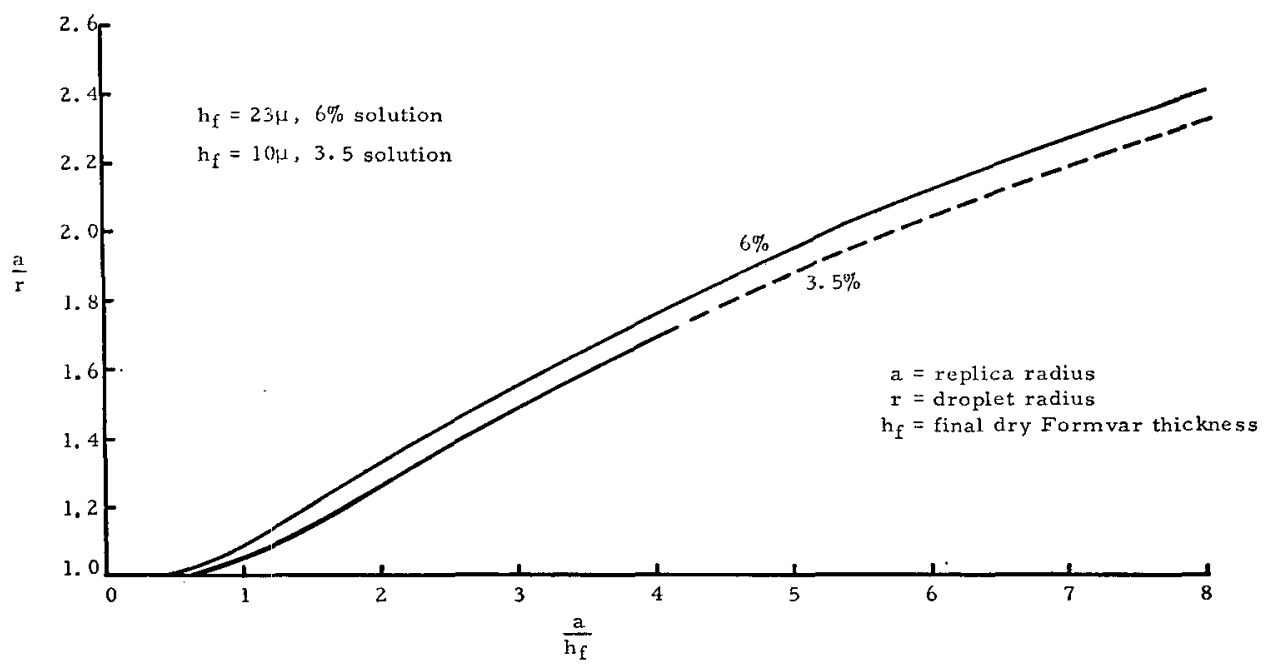

FIG. 9. Calibration factor related to dry Formvar thickness. 


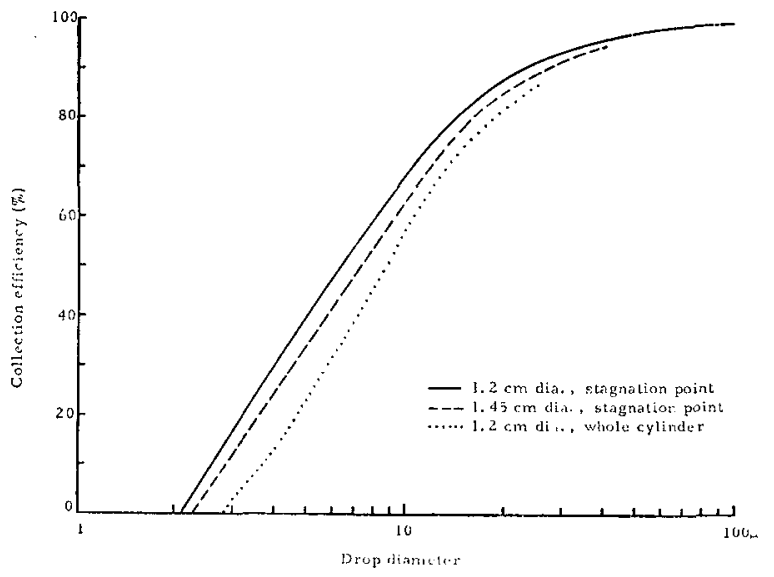

FIG. 10. Collection efficiency for droplets impacting on a cylinder. Curves representative of conditions at Flagstaff in summer cumulus clouds: true air speed $50 \mathrm{~m}$ per sec, temperature $0 \mathrm{C}$, pressure $500 \mathrm{mb}$.

are counted near the center of the film, near the stagnation point. When low concentrations of ice crystals are being detected, sometimes a much greater width of the film is used to increase the sampling volume. Ice crystals do not have spherical shapes and so for them a simple collection efficiency curve such as on Fig. 10 is not appropriate. However, by far the majority of ice crystals in natural clouds have sizes for which the collection efficiency is greater than 0.8 , and so the exact curve used for them is not important.

Fig. 10 indicates a practical lower limit of $3 \mu$ diameter for droplets collected by the units as presently used. Fig. 5 shows particles at about this size limit. A smaller diameter collector or higher air speed would permit small particles to be collected; 1 to $2 \mu$ diameter seems a practical limit for future designs.

The large end of the size range depends primarily on breakup during collection. Ice crystals of $100 \mu$ may be cracked and $200 \mu$ crystals broken (see Fig. 5); ice needles as long as $600 \mu$ have been collected unbroken. When a graupel pellet hits the film the replica has the appearance of a snowball which has struck a windshield; the number of graupel pellets can be counted, but the size of each is difficult to interpret.

Circular droplet replicas of even $150 \mu$ diameter have been found on the film. The size of the corresponding droplets is not known, because the size correction factor for such extremes has not been found, but possibly the impinging droplet was on the order of $50 \mu$ diameter. In the ground measurements in France (MacCready, 1962) replicas larger than $25 \mu$ diameter were not completely encapsulated, leaving a hole in the replica center. The maximum size having complete encapsulation must vary with concentration, but in any case the correction factor for the largest droplets will probably be somewhat indeterminate. Rupe ${ }^{6}$ found that the formula:

$$
\text { diameter (microns) }=\frac{1725}{\text { impact speed }\left(\mathrm{m} \mathrm{sec}^{-1}\right)}
$$

gives the largest size of a droplet which would not break on penetrating a kerosene surfact. At $50 \mathrm{~m}$ per sec this corresponds to a diameter of $34.5 \mu$. Weickmann (1953) found the constant in the above formula to be increased by an order of magnitude for collection in castor oil. From considering and viewing meny of the records from the continuous sampler, it seerrs likely that droplets under about $50 \mu$ diameter are unbroken, and perhaps this is true even for substantially larger droplets.

\section{Data reduction}

It has turned out to be most convenient to analyze the film by projecting it on a screen with a modified version of a standard stop-motion projector. For counting large particles such as big snow crystals and graupel the standard 16-mm projection It:ns system is used. For counting and examining small particles the lens system is replaced by a tube with the optics system from a microscope, usually set at 500 or 1000 power.

The main problem is to cope with the large amount of data generated each flight. At thie present stage of development of the system, automatic data reduction of these replica records does not yet seem justified. Some of the droplet records are of a quality for which automatic counting and sizing would he possible, but manual methods can give concentration; and spectra quickly and without intricate apparatus. Human interpretation is necessary during data reduction of complex snowflake replicas and of poor quality droplet replicas.

The film records are first exarnined crudely by running them through at 16 frames per second. The eye and mind react quickly enough to ascertain the general distribution of drop sizes, the preseace of ice crystals and graupel and the general quality of record.

The next step for general cloud studies is to get the concentrations of droplets and crystals and the general size ranges. These counts can be made rapidly from representative frames. The droplet counts must be corrected for droplet distortion, and then the collection efficiency factor used to determir.e true concentration. These corrections are especially q ick to apply when the droplet spectra are narrow. To facilitate correlating these data to the other cloud paremeters recorded on an analog chart during the airplane raverse, it has proved convenient to plot the data on a chart driven from a gear on the projector. The gear ratio is adjusted to put the particle information on the time scale of the cloud parameter records. When the sar.upler is run in the aircraft, three event markers on the cloud parameter chart

${ }^{6}$ Rupe, J. H., 1950: Critical impact relocities of water droplets as a problem in injector-spray sampling. Prog. Report \# 4-80, Jet Propulsion Lab., Calif. Inst. of Tec.., Sept. 
record every 1, 10 and 100 rotations of an idler wheel on the film. Thus the sampler data can easily be exactly correlated to the cloud data.

For detailed case studies complete size spectra and accurate concentrations are required. A data compilation technique worked out by the second author which has proved simple and effective is to move a strip of paper around the projected frame, putting the strip's left edge on the left edge of each replica image and making a mark on the strip at the right edge of the replica image. Then the dots are counted in the size categories, to provide spectra after the droplet distortion and collection efficiency corrections are applied. Rather than recording complete spectra it is often satisfactory to record only the concentration and the mean and standard deviation of the distribution. Obtaining the standard deviation is facilitated by putting a micron scale vertically on the strip (perpendicular to the droplet size scale which goes horizontally from left to right). Then slide a probability scale down the strip, with the 50 per cent cumulative probability line matching the horizontal position of the median droplet. Mark the strip at drop sizes corresponding to various cumulative probabilities, and make a best fit line through these points. Read off the micron size of one standard deviation ( 50 per cent to 84 per cent or 50 per cent to 16 per cent cumulative probability).

\section{Conclusions and recommendations}

The unique value of the continuous Formvar collection principle has been established over the last three years of development. In its most efficient form, it collects solid and liquid particles in a manner which permits relatively easy analysis. Some collection scheme such as this seems to be the only satisfactory approach to measuring small ice crystals on cloud physics research programs; the shape of the crystals is desired, as well as size (which must be defined with respect to shape) and concentration. For droplet measurements, the Formvar technique is good but some other optical method is a possibility. The optical method, light scattering or light shadow, measures the droplets in the air, avoiding some collection efficiency problems as well as avoiding droplet distortion and breakup on a surface; also in some cases the data acquisition is electronic and so further data reduction is unnecessary. The continuous collector is simpler than optical methods, and convenient to carry in small vehicles. In summary, the measurement of ice crystals apparently requires the continuous collector and the same device is effective for droplet measurements although competitive systems are a possibility.

The most basic problem with the continuous sampler is that, although in final form the device is relatively simple, it is based on many complex factors and interrelationships (nonlinear viscosity and surface tension variations in coating and encapsulations, various contradictory requirements for rapid evaporation of solvent without blushing or crystal melting, etc.). From the development studies it is apparent that all of these practical problems are solvable, because all have been solved at one time or another. The difficult matter is to find a compromise which solves them all simultaneously over a broad range of atmospheric situations with adjustments which are not impractically critical.

Experience with the present units of course has created ideas of how future designs can be improved. One suggestion to make the design requirements less critical is to give the film two separate parallel coatings, one of a type optimized for droplet collection, the other optimized for crystal collection. It would appear that the technique of vapor softening of precoated film warrants further consideration.

Any system, whether substantially new or merely an adaptation of the present one, requires a series of systematic tests at various air speeds and ambient temperatures with various solution concentrations, with various amounts of drying heat, for collecting both crystals and droplets. A final version, if made to cover a wide meteorological range, will have controllable heaters (initially controlled manually, later automatically).

The simple counting techniques associated with projecting the film by a stop-motion 16-mm projector and counting by hand have worked well. Even quantitative measurements take only a few minutes per frame, a time which is so short that the development of automatic techniques to cut down the time is hardly justified now.

The absolute calibration technique described in this report shows that one of the primary questions pertaining to this collector method has been solved in principle. More detailed calibrations using the same method should be made, including tests at various temperatures and Formvar concentrations.

Acknowledgments. The initial development of the continuous sampler and the continuity for its continued development were made possible by the support extended to the Atmospheric Research Group by the Atmospheric Sciences Section of the National Science Foundation (Grant 11969). The laboratory studies and development of the latest unit were sponsored by the Naval Research Laboratory under Contract Nonr-3819(00)(X). Mr. Robert Ruskin of NRL has been particularly helpful in the cooperative phases of the work. The program has been materially aided by concurrent sponsorship of cloud physics studies at MRI, using and developing this type of sampler, by the U. S. Army Electronics Research and Development Laboratory (Contract DA 36-039 SC-89066). Some of the development was sponsored by MRI internal research funds.

Many ARG-MRI people have participated actively in the development of the continuous droplet sampler. Mr. J. A. K. Lake handled the development of the early versions of the sampler; Mr. R. Sloane developed the computer program associated with the theoretical distortion calculations and did much of the laboratory work on materials; Mr. E. E. Hindman, II, aided in 
the laboratory investigations and had primary responsibility for the operation of the collectors at Flagstaff in 1962 and 1963; Mr. R. Williamson completely handled the absolute calibration procedure and the design of the latest mechanical unit.

\section{REFERENCES}

Farlow, N. H., and F. A. French, 1956: Calibration of liquid aerosol collectors by droplets containing uniform size particles. $J$. colloidal Sci., 11, 177-183.

Hindman, E. E., II, 1964: Continuous sampler for settling particles. $J$. de Recherches almosphériques, I., 29-35.
MacCready, P. B., Jr., 1962: The continuous particle sampler at the Puy de Dome comparison con erence. Bull. Obs. du Puy de Dome, 1, 19-30.

Schaefer, V. J., 1956: Preparation of snow crystal replicas-VI. Weatherzise, 9, 132-135.

-., 1962: The vapor method for mating replicas of liquid and solid aerosols. J. appl. Meteor., 3, +113-418.

Todd, C. J., 1961: A study of cloud composition. Proc. Ninth Wea. Radar Conf., Boston, Amer. Meteor, Soc., 280-285.

-_, 1964: Ice crystal development iı a seeded cumulus cloud. $J$. atmos. Sci., in press.

Weickmann, H. K., and H. J. aufn Kampe, 1953: Physical properties of cumulus clouds. $J . N^{\prime}$ eteor., 10, 204-211. 